\title{
Unterstützung gemeinschaftlicher Projekte zur Wärmeversorgung
}

\author{
Eine gemeinschaftliche Wärmeversorgung \\ bietet große Potenziale. Konventionelle Mach- \\ barkeitsstudien stoßen bei derartigen Projekten \\ häufig an ihre Grenzen. Hier setzt ein neues \\ Entscheidungsmodell zur Entwicklung von \\ Wärmeversorgungskonzepten an. \\ Von Volker Ruwisch, Nico Michalak, Anke Daub \\ und Folker Roland
}

m Fokus der öffentlichen Wahrnehmung zur Energiewende steht in der Regel die Stromproduktion. Es wird vor allem diskutiert, wie Kernenergie und fossile Brennstoffe durch erneuerbare Energieträger ersetzt werden können. Der Stromanteil am Endenergieverbrauch beträgt allerdings nur circa 22 Prozent, wohingegen die Wärme mit fast 50 Prozent die deutlich größere Bedeutung hat.

Von dem gesamten Endenergieverbrauch in Höhe von circa 2.424 Terrawattstunden (TWh) in 2011 entfiel der größte Anteil von circa 1.180 TWh auf die Wärme (Raum- und Prozesswärme). Für Strom wurden circa 525 TWh genutzt sowie circa 719 TWh für Kraftstoffe. Mit Blick auf den Klimawandel hat der Wärmebereich deshalb eine große Relevanz; dies gilt umso mehr, als der Anteil der erneuerbaren Energieträger im Wärmebereich mit 10,2 Prozent deutlich niedriger ist als im Strombereich mit 23,5 Prozent (Stand 2012). Die Umstellung der Wärmeversorgung, insbesondere im Altbaubestand mit den oft sehr hohen Verbräuchen, erscheint für eine zeitnahe Reduktion von $\mathrm{CO}_{2}$ Emissionen deshalb dringend geboten.

Des Weiteren werden sehr viele Energieträger (zum Beispiel Heizöl, Erdgas) unnötig für die Wärmebereitstellung eingesetzt, weil die bei der Stromproduktion als Kuppelprodukt auch anfallende Wärme häufig nicht genutzt, sondern über Kühltürme in die Atmosphäre abgegeben wird. Bei durchschnittlichen elektrischen Wirkungsgraden der konventionellen fossilen Kraftwerke von circa 40 bis 45 Prozent könnte ein großer Teil des Raumwärmebedarfs durch die anfallende „Abwärme“ der Stromproduktion gedeckt werden.

Unabhängig von dem Ziel der Ausschöpfung möglicher Einsparpotenziale zur Reduzierung des Wärmebedarfs an sich (zum Beispiel durch Dämmmaßnahmen) bieten daher die Steigerung der Energieeffizienz durch Kraft-Wärme-Kopplung einerseits und der verstärkte Einsatz von erneuerbaren Energieträgern andererseits beachtliche Möglichkeiten zu einer zeit- nahen Verringerung klimaschädlicher Emissionen von über 50 Prozent. Dies lässt sich insbesondere im ländlichen Raum durch die Verstromung der dort verfügbaren Biomasse bei gleichzeitiger Wärmenutzung (zum Beispiel in Biogasanlagen mit angeschlossenen Blockheizkraftwerken) vergleichsweise problemlos realisieren.

\section{Herausforderung: Klimaverträgliche Wärmeversorgung}

Um über Wärmenetze den Endverbrauchern zu vertretbaren Preisen Wärme anbieten zu können, ist es vorteilhaft, wenn diese nicht weit transportiert werden muss. Deshalb lassen sich Nahwärmekonzepte idealerweise dann umsetzen, wenn Großabnehmer oder eine verdichtete Bebauung vorhanden sind. Da dies in ländlichen Gebieten oft nicht der Fall ist, stellt die wirtschaftliche Verteilung der Wärme über derartige Nahwärmenetze eine planerische Herausforderung dar.

Zusätzlich zu den nicht optimalen Siedlungsstrukturen in Dörfern mit weit auseinanderliegenden potenziellen Anschlussobjekten (Haushalten, Betrieben, öffentlichen Gebäuden) kann aufgrund der fehlenden Verpflichtung, sich an ein Nahwärmenetz anschließen zu lassen, nicht davon ausgegangen werden, dass sich eine hohe Anschlussdichte problemlos erreichen lässt. Da die Gebäude bereits bewohnt sind und deshalb in der Regel über eine funktionierende Heizungsanlage verfügen, ist die Bereitschaft, diese zu ersetzen, stark vom Alter und dem Zustand der jeweiligen Heizung abhängig. Dies hat zur Konsequenz, dass der Zeitpunkt der geplanten Installation des Nahwärmenetzes nur selten mit der Entscheidung über eine Erneuerung des Heizungssystems eines einzelnen Haushaltes zusammenfällt. Bei Neubaugebieten, in denen ohnehin über das zu installierende Heizungssystem entschieden werden muss, ist dies dagegen nicht der Fall. Um trotzdem eine hohe Anschlussdichte gerade im Altbaubestand zu erreichen, ist es deshalb aus Sicht der Wärmekunden essenziell, dass die neue Wärmeversorgung deutliche ökonomische Vorteile gegenüber der bestehenden aufweist. Dies setzt voraus, dass für das geplante Nahwärmenetz eine wirtschaftlich tragfähige Lösung gefunden wird.

Angesichts der Existenz zahlreicher Biogasanlagen sowie der dort anfallenden und häufig nicht sinnvoll genutzten Wärme bieten gerade ländliche Regionen grundsätzlich ein großes Potenzial für klimaverträgliche Wärmebereitstellungskonzepte. Die Nutzung dieser Wärme kann zum Beispiel in Form eines von der Dorfgemeinschaft getragenen Bioenergie- 


\author{
„Das Planungsinstrument \\ kann die mit der \\ sozialen Dynamik \\ verbundenen Schwierigkeiten \\ auffangen."
}

dorfkonzeptes erfolgen. Hier wird durch die Dorfbevölkerung versucht, im Rahmen eines gemeinschaftlichen Diskussions-, Planungs- und Entscheidungsprozesses ein sozial und wirtschaftlich tragfähiges Wärmeversorgungskonzept zu entwickeln. Dieses geschieht im Rahmen von Befragungen, Workshops, Arbeitsgruppen und Dorfversammlungen und erstreckt sich deshalb zwangsläufig über einen längeren Zeitraum, der durchaus mehrere Jahre betragen kann, was häufig damit einher geht, dass sich die Planungsparameter im Zeitverlauf verändern. So kann es sein, dass vorher anschlusswillige Hauseigentümer das Interesse verlieren, während andere neu hinzukommen.

Bei der Betreuung von Bioenergiedorfprojekten wurde festgestellt, dass konventionelle Machbarkeitsstudien, die in der Regel von Ingenieurbüros erstellt werden, meist nicht die für derartig komplexe Gemeinschaftsprojekte notwendige Flexibilität aufweisen, da sie ein Nahwärmekonzept nur für eine konkrete Datenkonstellation entwickeln. Bei Veränderungen dieser Daten als Folge der sozialen Dynamik sind jeweils Neuberechnungen erforderlich, die sich nur mit einem erheblichen zeitlichen und damit finanziellen Mehraufwand durchführen lassen. Gerade in Bioenergiedorfprojekten sind die zumeist ehrenamtlich tätigen Personen aber nur begrenzt bereit, in finanzielle Vorleistung zu treten, solange ein positiver Projektausgang nicht sicher ist.

Hieraus lässt sich als Anforderung an das einzusetzende Planungsinstrumentarium ableiten, dass es nicht nur in der Lage sein muss, ein wirtschaftlich optimales Nahwärmekonzept zu ermitteln, sondern auch flexibel auf die im Zeitablauf eintretenden Veränderungen (zum Beispiel bezüglich der Anzahl und der Verteilung der Anschlussobjekte) zu reagieren. Aufgrund der großen Bedeutung wirtschaftlich, ökologisch und sozial tragfähiger Wärmebereitstellungskonzepte für eine klimaverträgliche Energieversorgung wurde im Rahmen eines interdisziplinären Forschungsprojektes („Nachhaltige Nutzung von Energie aus Biomasse im Spannungsfeld von Klimaschutz, Landschaft und Gesellschaft“) an der Georg-August-Universität Göttingen ein diesen Anforderungen genügendes Planungstool entwickelt. Auf der Basis eines Entscheidungsmodells lässt sich damit nicht nur der kostengünstigste Verlauf eines Nahwärmenetzes planen und dessen Wirtschaftlichkeit beurteilen, es können darüber hinaus auch im Zeitablauf sich verändernde Vorgaben zu Anschlussobjekten oder konkreten Streckenverläufen nachträglich einbezogen und im Hinblick auf ihre ökonomischen Auswirkungen zeitnah analysiert werden. Als Optimalitätskriterium wird die ökonomische Zielgröße des Kapitalwerts herangezogen. Soziale und ökologische Aspekte werden über Nebenbedingungen im Modell berücksichtigt und beeinflussen dadurch unmittelbar das Ergebnis.

\section{Entwicklung eines Nahwärmekonzepts für ein Bioenergiedorf}

Das im Rahmen des Forschungsprojektes entwickelte Tool wurde zur Planung eines Nahwärmenetzes in einem niedersächsischen Ort eingesetzt und validiert. In diesem Ort mit circa 650 Einwohnern und etwa 150 Wohngebäuden war bereits eine Biogasanlage mit einer Leistung von $500 \mathrm{~kW}_{\mathrm{el}}$ installiert, deren Abwärme bisher nicht sinnvoll genutzt wurde und für die daher in Zusammenarbeit mit der Dorfgemeinschaft ein Wärmekonzept entwickelt werden sollte. Die Planung beschränkte sich zunächst auf den südlichen Ortsteil, in dem 38 Haushalte bei Befragungen ihre Bereitschaft geäußert hatten, sich an das Nahwärmenetz anschließen zu lassen. Während sich die Biogasanlage im alleinigen Eigentum des Landwirts befindet, wurde für den Betrieb des Nahwärmenetzes eine Bürgergenossenschaft gegründet, für die laufende Betriebskosten (Personal, Versicherung etc.) zu berücksichtigen sind. Da durch die Nutzung der Abwärme der Biogasanlage die Wärmeversorgung der anschlusswilligen Haushalte in den Wintermonaten nicht vollständig gewährleistet werden kann, ist es ferner notwendig, einen Spitzenlastkessel zu installieren, der ebenfalls von dem Landwirt errichtet und betrieben wird. Es ist geplant, dass die Bürgergenossenschaft die Wärme (Abwärme und Spitzenlast) zu einem festen einheitlichen Preis von dem Landwirt bezieht und sie an ihre Mitglieder als Wärmekunden verkauft.

Innerhalb des langen Planungszeitraums veränderten sich die Zusammensetzung und damit die räumliche Verteilung der Anschlussobjekte mehrfach. Dies und spezifische örtliche Gegebenheiten, die erst bei der Detailplanung deutlich wurden (Probleme bei einer Gewässerquerung sowie mögliche erweiterte Auflagen bei der Verlegung der Leitungen im Bereich einer Bundesstraße), führten dazu, dass im Projektverlauf wiederholt eine Neuerstellung der ursprünglichen Machbarkeitsstudie notwendig gewesen wäre. Stattdessen kam das entwickelte Planungstool zum Einsatz, mit dem die Bürgergenossenschaft bei ihren anstehenden Entscheidungen unterstützt werden konnte.

In diesem Tool wird die Planungssituation mithilfe eines linearen Optimierungsmodells abgebildet, das aus einer Zielfunktion sowie Variablen und Nebenbedingungen besteht. Als Beurteilungskriterium wird in der Zielfunktion mit dem Kapitalwert gearbeitet, der sich auf einen Zeitraum von 20 Jahren bezieht und in den als positive Komponenten die Umsatzerlöse aus dem Wärmeverkauf sowie gegebenenfalls staatliche 
Zuschüsse einfließen und als negative Bestandteile insbesondere die Auszahlungen für den Bau des Nahwärmenetzes. Die Höhe dieser zahlungsrelevanten Kosten hängt dabei nicht nur von der Länge des einzelnen Netzteilstückes ab, sondern auch davon, wie aufwendig die notwendigen Verlegearbeiten sind (Teerdecke, Feldweg, Gartenland). Alle relevanten Eingabeparameter (zum Beispiel Wärmebedarfsmengen der Haushalte, Länge und Kostensätze der Netzteilstücke) sind im Vorfeld zu erfassen und können in der Regel aus dem Datenmaterial der Machbarkeitsstudie abgeleitet werden; gegebenenfalls sind noch in geringem Umfang Nacherfassungen erforderlich.

$\mathrm{Zu}$ entscheiden ist darüber, welche der anschlussbereiten Objekte in die Nahwärmeversorgung aufgenommen werden und über welchen Netzverlauf, durch Auswahl der hierfür notwendigen Teilstücke, diese kostengünstig angeschlossen werden können. Mithilfe von Nebenbedingungen wird sichergestellt, dass ein zusammenhängendes Netz entsteht und alle Anschlussobjekte erreichbar sind.

Unter Einsatz der Software XPress IVE wird dann für dieses gemischt ganzzahlige lineare Optimierungsproblem zunächst das ökonomisch vorteilhafteste Versorgungsnetz im Hinblick auf die Anschlussobjekte und den konkreten Netzverlauf identifiziert. Dieser Vorschlag (Variante A) und der maximal erreichbare Kapitalwert (in diesem Fall in Höhe von 110.694 Euro) bilden die Basis für weitergehende Analysen. Dabei hat die Berechnung ergeben, dass fast alle Haushalte an das ermittelte optimale Nahwärmenetz angeschlossen werden; nur einige Objekte werden nicht berücksichtigt, weil sie aufgrund langer Zuleitungen hohe Anschlusskosten verursachen, die durch die Wärmeerlöse nicht kompensiert werden. Infolge der hohen spezifischen Verlegekosten auf der Bundesstraße wird diese nur für ein Netzteilstück genutzt.

\section{Analyse von Planungsvarianten}

Der Vorschlag für den Verlauf des Nahwärmenetzes ergibt sich, wenn man der Entscheidung allein wirtschaftliche Aspekte zugrunde legt. Da aber gerade im dörflichen Zusammenleben soziale Beziehungen eine große Bedeutung haben und es für derartige Projekte wichtig ist, dass sie von der Dorfgemeinschaft möglichst umfassend getragen werden, ist im Einzelfall abzuwägen, ob nicht von der wirtschaftlichen Optimallösung bewusst abgewichen werden sollte, indem auch unwirtschaftliche Anschlussobjekte in das Netz integriert werden. Durch eine Veränderung der Nebenbedingungen lässt sich die-

\begin{tabular}{llc}
\hline Variante & Beschreibung & Kapitalwert \\
\hline A & freies Optimum & $110.694 €$ \\
\hline B & $\begin{array}{l}\text { alle potenziellen Hausobjekte werden ans Netz } \\
\text { angeschlossen }\end{array}$ & $88.007 €$ \\
\hline C & $\begin{array}{l}\text { für den Netzverlauf soll die Bundesstraße } \\
\text { nicht genutzt werden }\end{array}$ & $91.744 €$ \\
\hline D & Kombination der Varianten B und C & $65.096 €$
\end{tabular}

Tabelle 1: Planungsvarianten ses leicht in das Entscheidungsmodell einbeziehen und über eine Variationsrechnung die für diese neue Konstellation dann wirtschaftlich beste Lösung mit einem gegebenenfalls veränderten Netzverlauf berechnen (Variante B). Bezogen auf die drei oben genannten Haushalte ergibt sich eine Kapitalwertverschlechterung in Höhe von circa 20 Prozent. Da der Kapitalwert nach wie vor im positiven Bereich liegt und die Differenz in Höhe von 22.687 Euro angesichts des langen Planungszeitraums von 20 Jahren vergleichsweise gering ist, erscheint dieser wirtschaftliche Nachteil zugunsten der Förderung des Gemeinschaftsgefühls im Dorf in diesem Fall vertretbar.

Eine weitere Variante ergibt sich daraus, dass für die Nutzung der Bundesstraße hohe Auflagen (wie zum Beispiel Gewährleistungsverpflichtungen) in der Diskussion sind. Aus diesem Grund erscheint es sinnvoll zu analysieren, welche Auswirkungen ein Verzicht auf die Nutzung der Bundesstraße hätte (Variante C). Eine entsprechende Umsetzung bei der Modellformulierung und eine Optimierung führen in der neuen Lösung zu einem veränderten Netzverlauf sowie zu einer Reduzierung des Kapitalwerts um circa 17 Prozent gegenüber der Ausgangslösung (Variante A).

Eine gleichzeitige Berücksichtigung der Vorgaben der Varianten B und C (Anschluss aller potenziellen Haushalte und Vermeidung der Nutzung der Bundesstraße) vermindert den Kapitalwert weiter. Er unterscheidet sich von dem der Ausgangslösung um 41 Prozent und der zugehörige Netzverlauf greift an mehreren Stellen auf alternative Teilstrecken zurück.

Die einzelnen Varianten sowie die für sie ermittelten Kapitalwerte sind in Tabelle 1 zusammengefasst. Die Tatsache, dass die Kapitalwerte positiv sind, verdeutlicht, dass sich alle Entscheidungsalternativen wirtschaftlich vorteilhaft umsetzen lassen.

\section{Grenzen der Wirtschaftlichkeit}

Für die Bürgergenossenschaft ist ferner von großem Interesse, welches wirtschaftliche Risiko mit derartigen Projekten verbunden ist. Ein Indiz hierfür ist die absolute Höhe des jeweiligen Kapitalwertes. Da die oben ermittelten Ergebnisse einen deutlichen Abstand zu dem kritischen Wert von Null (Grenze zur Unwirtschaftlichkeit) aufweisen, ist das Risiko als vergleichsweise gering einzuschätzen. Wie an dem Kapitalwert ersichtlich ist, können sogar Entwicklungen wie beispielsweise eine Erhöhung des Wärmeeinkaufspreises oder ein Rückgang der Wärmeabnahmemengen (zum Beispiel durch Dämmmaßnahmen) in gewissen Grenzen aufgefangen werden, ohne die Wirtschaftlichkeit des Projektes zu gefährden.

Für das vorgestellte Beispiel wurden entsprechend die Auswirkungen einer Erhöhung des Wärmeeinkaufspreises analysiert. Ausgehend von der Variante D (keine Nutzung der Bundesstraße und Anschluss aller interessierten Wärmekunden) hat eine Sensitivitätsanalyse ergeben, dass bei einer Erhöhung um circa 0,5 Cent pro Kilowattstunde $(\mathrm{kWh})$ und damit einer Verringerung der Handelsspanne von 5,7 auf 5,2 Cent/kWh der 
Kapitalwert auf null sinkt und damit die Grenze der Wirtschaftlichkeit erreicht ist. Dies bedeutet, dass eine mögliche Preiserhöhung in größerem Ausmaß nur durch eine entsprechende Anpassung des Wärmeverkaufspreises aufgefangen werden kann, will man den wirtschaftlichen Betrieb nicht gefährden. Da davon auszugehen ist, dass der Heizölpreis im Projektverlauf steigen wird, bestehen dazu in der Zukunft auch Spielräume, ohne die wirtschaftliche Vorteilhaftigkeit des Nahwärmebezugs aus der Sicht der Wärmeabnehmer (im Vergleich zu einer konventionellen Heizung) infrage zu stellen.

Analysiert man auf der Basis des ursprünglichen Wärmeverkaufspreises und der Konstellation in Variante D das Risiko einer Unwirtschaftlichkeit aufgrund einer geringeren Wärmeabnahmemenge als gedacht, so lassen sich weitere Aussagen zur Stabilität der oben ermittelten Lösung treffen. Ursachen für einen derartigen Nachfragerückgang könnten beispielsweise in den oben bereits angesprochenen zusätzlichen Dämmmaßnahmen, Wegzug oder anders begründeten Leerständen von Anschlussobjekten liegen. Die jährliche Wärmeabnahmemenge in Variante D beträgt in der Summe durchschnittlich circa $937.000 \mathrm{kWh}$. Eine diesbezügliche Sensitivitätsanalyse ergibt, dass bis zu einem Nachfragerückgang von circa neun Prozent die Wirtschaftlichkeit des Gesamtprojektes erhalten bleibt.

Wie die oben dargestellten Varianten und die Sensitivitätsanalyse verdeutlichen, lassen sich Vorgaben mithilfe des Planungstools im Modell problemlos berücksichtigen. Die Ergebnisse können damit den Meinungsbildungsprozess der Dorfgemeinschaft wirkungsvoll unterstützen, sodass die Entscheidung über die Realisierung des Projekts, den Verlauf des Nahwärmenetzes und die Auswahl der Anschlussobjekte fundiert und zeitnah getroffen werden kann.

\section{Fazit}

Die Ausführungen machen deutlich, dass gemeinschaftliches Engagement bei der Wärmeversorgung zum einen zu einer deutlichen und schon kurzfristig erreichbaren Verbesserung der Klimaverträglichkeit führen kann. So lassen sich selbst in dem vergleichsweise kleinen untersuchten Ortsteil mit dem neuen Wärmekonzept circa 100.000 Liter Heizöl im Jahr einsparen. Zum anderen werden durch solche dezentralen Ansätze regionale Wirtschaftskreisläufe gestärkt.

Die bei Gemeinschaftsprojekten häufig langwierige Meinungsbildung und Entscheidungsfindung stellen eine besondere Herausforderung an die einzusetzenden Planungsmethoden dar. Das in dem Forschungsprojekt entwickelte und oben beschriebene Planungstool kann durch seine große Flexibilität die mit der sozialen Dynamik verbundenen Schwierigkeiten auffangen und - prozessbegleitend eingesetzt - die Expertise von Ingenieurbüros ergänzen. Die im Rahmen der Modellierung durchführbaren Variationsrechnungen und Sensitivitätsanalysen ermöglichen es, wirtschaftlich tragfähige Nahwärmenetzkonzepte $\mathrm{zu}$ identifizieren, aber auch die Grenzen ihrer Wirtschaftlichkeit aufzuzeigen.

\section{Literatur}

Bundesministerium für Umwelt, Naturschutz und Reaktorsicherheit (2012): Entwicklung der Erneuerbaren Energien in Deutschland im Jahr 2011, Berlin.

Bundesministerium für Wirtschaft und Technologie (2013): Zahlen und Fakten - Energiedaten, Berlin.

Daub, A./Uhlemair, H./Ruwisch, V./Geldermann, J. (2013): Optimising Bioenergy Villages' Local Heat Supply Networks. In: Ruppert, H./ Kappas, M./lbendorf, J. (Hrsg.): Sustainable Bioenergy Production An Integrated Approach. Dordrecht u. a. S. 219-238.

Götze, U. (2008): Investitionsrechnung, 6. Auflage. Berlin, Heidelberg. Hirschl, B./Aretz, A./Prahl, A./Böther, T./Heinbach, K./Pick, D./Funcke, S. (2010): Kommunale Wertschöpfung durch Erneuerbare Energien, Schriftenreihe des IÖW 196/10. Berlin.

Ruppert, H./Schmuck, P./Eigner-Thiel, S./Girschner, W./KarpensteinMachan, M./Ruwisch, V./Sauer, B./Roland, F. (2008): Das Bioenergiedorf - Voraussetzungen und Folgen einer eigenständigen Wärmeund Stromversorgung durch Biomasse für Landwirtschaft, Ökologie und Lebenskultur im ländlichen Raum. Göttingen.

Ruppert, H./Eigner-Thiel, S./Girschner, W./Karpenstein-Machan, M./ Roland, F./Ruwisch, V./Sauer, B./Schmuck, P. (2008): Wege zum Bioenergiedorf - Leitfaden. Göttingen.

Uhlemair, H. (2012): Optimierung des Produktions- und Distributionssystems von Bioenergiedörfern. Göttingen.

Werners, B. (2007): Kurzeinführung in XPress-IVE - Standardsoftware zur Lösung linearer, kontinuierlicher und (gemischt-)ganzzahliger Programmierungsmodelle. Bochum.

AUTOREN + KONTAKT

Dipl.-Kfm. Volker Ruwisch ist Vertretungsprofessor für Logistikmanagement an der Hochschule Harz Wernigerode und wissenschaftlicher Mitarbeiter am Interdisziplinären Zentrum für nachhaltige Entwicklung der Universität Göttingen (IZNE).

Hochschule Harz, Friedrichstr. 57-59,

38855 Wernigerode. E-Mail: vruwisch@hs-harz.de

Dipl.-Wirt.-Inf. Nico Michalak ist wissenschaftlicher Mitarbeiter am Interdisziplinären Zentrum für nachhaltige Entwicklung der Universität Göttingen (IZNE).

Universität Göttingen, Goldschmidtstr. 1, 37077 Göttingen. E-Mail: nmichal@gwdg.de

Dr. Anke Daub ist wissenschaftliche Mitarbeiterin am Lehrstuhl für Produktion und Logistik der Universität Göttingen.

Universität Göttingen, Platz der Göttinger Sieben 3, 37073 Göttingen. E-Mail: adaub@gwdg.de

Dr. Folker Roland ist Professor für Betriebswirtschaftslehre, insbesondere Produktions- und Logistikmanagement, an der Hochschule Harz Wernigerode.

Hochschule Harz, Friedrichstr. 57-59, 38855 Wernigerode. E-Mail: froland@hs-harz.de
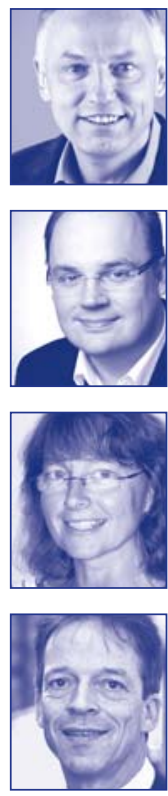